Bull. Mater. Sci., Vol. 21, No. 6, December 1998, pp. 479-484, (C Indian Academy of Sciences.

\title{
Spectroscopic studies of hydrogen related defects in CVD diamond
}

\author{
M PANDEY*, V SUGANDHI, R D'CUNHA, A K SIKDER ${ }^{\dagger}$ and D S MISHRA ${ }^{\dagger}$ \\ Spectroscopy Division, Bhabha Atomic Research Centre, Mumbai 400085 , India \\ tDepartment of Physics, Indian Institute of Technology, Mumbai 400 076, India
}

MS received '13 May 1998

\begin{abstract}
Thin diamond films prepared by the hot filament chemical vapour deposition (HFCVD) method at various deposition pressures have been characterized using a variety of spectroscopic techniques. Interpretation of the spectral details have provided useful information about the nature of the films. Deposition pressure appears to affect the quality of the diamond films which is reflected in terms of the position and width of the characteristic Raman peak of diamond. Raman spectra of the films prepared at low deposition pressures showed the presence of a sharp peak at $\sim 1332 \mathrm{~cm}^{-1}$ characteristic of the $T_{2 \mathrm{~g}}$ mode of diamond. The study of the effect of deposition pressure on the diamond growth, shows that in the range between 20 torr and 60 torr, there is little effect on the width and the shift of the $1332 \mathrm{~cm}^{-1}$ Raman peak. However, at higher pressures the peak showed a blue shift and was considerably broadened. These studies indicate the development of strain in the lattice due to the introduction of unetched hydride layer, at higher deposition pressures, as well as distortions in the lattice leading to partial lifting of the degeneracy of the $T_{2 \mathrm{~g}}$ mode. A broad band corresponding to the non-diamond phase (which exists at the grain boundaries, interface or as inclusions inside the grain), which can be attributed to the effect of hydrogen impurity creeping into the lattice at higher deposition pressures is also observed. SEM and XRD patterns have confirmed the dominance of diamond phase in these films.
\end{abstract}

Keywords. CVD diamond; Raman spectra; hydrogen related defects.

\section{Introduction}

The recognition of the unique properties of diamond and its potential application as a structural material have revived interest in the growth and characterization of diamond films. Its mechanical strength finds application in wear resistant coatings, while its high thermal conductivity and electrical resistivity make it useful as a heat sink and high temperature semiconductor, respectively. It can also serve as an optical window because of its high optical transmission over a wide range of wavelengths.

Until recently, synthetic diamonds were grown under high pressure and high temperature with the addition of liquid metal catalysts to reduce the equilibrium pressure required for conversion of graphite to diamond (Liander and Lundblad 1960). Diamond synthesis by chemical vapour deposition (CVD) at low pressure and moderate temperatures was first achieved by Eversole (1962). Several techniques based on CVD for diamond growth have since been developed and have been extensively reviewed in literature (Angus and Haymann 1988; Backmann and Messier 1989; Spear 1989; Yarbrough and Messier 1990; Zhu et al 1991). These include

*Author for correspondence
HFCVD, microwave plasma, rf glow discharge plasma and electron assisted CVD and many others.

Precise characterization of CVD diamond coatings has become essential in view of their strong potential applications in industry. Raman scattering has emerged as a straightforward non-destructive tool for the identification of diamond structures and assignment of the phases of graphitic carbon, as well as hydrogen-rich tetrahedral carbon (diamond like carbon). Raman scattering is an inelastic scattering process that probes the vibrational modes of the material. As the material is strained, a change in the interatomic distances results in changes in the interatomic forces leading to a shift in the vibrational frequency. Thus, Raman scattering can be used to find out the average internal stress in the grains (Schadler and Galiotis 1995).

Diamond films grown by CVD contain a large variety of defects like interstitials, grain boundaries, stacking faults, twins and dislocations. The presence of these defects may limit their applications. To achieve good quality diamond films, it is essential to understand the origin of these defects. Many of the above defects are related to the presence of hydrogen in the film. Although hydrogen is essential for diamond growth, its presence in the film leads to deterioration of the quality of the film. Therefore, hydrogen incorporation and its role in 
determining the properties of CVD diamond is very critical. Many workers have studied the hydrogen impurities in CVD diamond films by various techniques, such as IR, NMR, EPR etc.

In this study the morphology and the crystal structure of the diamond films were studied by SEM and XRD, respectively. The hydrogen related defects were studied by Raman spectroscopy. These measurements are correlated with various deposition parameters of the growth process.

\section{Experimental}

Diamond films were prepared using the HFCVD technique. The chamber was evacuated by means of Rotary and diffusion pumps. A mixture of $0.8 \% \mathrm{CH}_{4}$ in hydrogen $\left(\mathrm{H}_{2}\right)$ was used as the precursor gas. The flow of the gases was monitored using mass flow controllers. A high current, low voltage a.c. transformer was used for heating the filament up to $2300^{\circ} \mathrm{C}$. A series of samples at different deposition pressures were prepared at the growth rate of $0.5-1.0 \mu \mathrm{m} / \mathrm{h}$. The substrates used in the present study were semiconductor grade $p^{+}$-silicon wafers with $\langle 100\rangle$ orientation. These substrates were treated with $6 \mu \mathrm{m}$ diamond paste prior to deposition. The substrates were heated to a temperature $(T s)$ of $925^{\circ} \mathrm{C}$, maintaining them at a distance of 5-6 mm away from the filament. A tiny thermocouple and/or an optical pyrometer were used to measure $T s$. The details of the experimental set up are given elsewhere (Sharda et al 1996).

Raman spectra of the films were recorded using the Ramalog (spex) double monochromator with $\mathrm{Ar}^{+}$laser $(\lambda=514.5 \mathrm{~nm}, 200 \mathrm{~mW})$ excitation. The scattered light from the film was collected in the back scattering geometry. The Raman signal was detected using a cooled PMT in the photon counting mode. The instrument was interfaced with a Personal Computer (PC) for data collection and processing. The Raman scattering signal for the sample grown at 80 torr pressure was found to be weak. Therefore, measurements for this sample were carried out by changing the excitation to the blue line $(\lambda=488 \mathrm{~nm})$ of the $\mathrm{Ar}^{+}$ion laser. Enhancement in the signal by a factor of five was observed.

\section{Results and discussion}

Figure 1 shows the typical SEM micrograph, which indicates mixed orientations of the crystallites. These pictures also show the sharp and clear facets indicating the good quality of the diamond crystallites. A typical XRD pattern of the films (figure 2) shows the presence of the diamond phase. Various reflections corresponding to (111), (400) and (311) planes of diamond are also observed, while the reflections from the (111) planes are found to be most prominent. These observations are further supported from the SEM pictures.

Raman scattering measurements were carried out from the various regions of the CVD diamond films in the spectral range $1250 \mathrm{~cm}^{-1}-1600 \mathrm{~cm}^{-1}$. The Raman spectra of the diamond films deposited at different deposition pressures (20-60 torr) of $\mathrm{CH}_{4}$ and $\mathrm{H}_{2}$ mixture are shown in figure 3. The characteristic peak at $1332 \mathrm{~cm}^{-1}$ corresponding to the $T_{2 \mathrm{~g}}$ mode of diamond is clearly seen in the figure. It can be seen that at low deposition pressures this peak is sharp and intense. The characteristic band due to the non-diamond phase is found to be almost absent, indicative of the fact that at low deposition pressures ( $\leq 60$ torr), the HFCVD growth leads to more of the diamond phase.

The inset (figure 3) shows the shift and width of the $1332 \mathrm{~cm}^{-1}$ Raman peak at different deposition pressures. As is seen clearly, there exists a range between 20 torr and 60 torr, where there is almost no shift in this peak. However, at higher deposition pressure ( 80 torr), this peak is blue shifted by $2.7 \mathrm{~cm}^{-1}$. Also, beyond a deposition pressure of 80 torr, the full width at half maximum (FWHM) of the Raman peak increases rapidly.

In order to estimate the correct parameters, a background, assumed to be due to luminescence, is subtracted, and the resultant (spectral plot) is fitted to a sum of a Lorentzian peak and three Gaussian peaks. The nondiamond contribution was observed as a broad band, which could be deconvoluted into bands at $1380 \mathrm{~cm}^{-1}$ (D-band) and bands centred at $1427 \mathrm{~cm}^{-1}$ and $1533 \mathrm{~cm}^{-1}$ (G-band), given in table 1. It has already been reported that the G-band consists of two broad features centred at $1470 \mathrm{~cm}^{-1}$ and $1550 \mathrm{~cm}^{-1}$ (Von Kaenel et al 1996).

Hydrogen is known to play an important role in the growth of diamond by CVD techniques. The addition and subtraction of the top growing layer of diamond takes place simultaneously. This accounts for the observed slow rate of diamond growth by the HFCVD technique. Actually, the growing layers have to be subsequently protected by atomic hydrogen $(\mathrm{H})$ (present in the vicinity of the hot filament), from getting converted into non-diamond form. The same $\mathrm{H}$ is also used to etch the top hydride layer (formed earlier in order to passivate non-diamond transformation) (Bar-Yam and Moustakas 1989; Fanciulli and Moustakas 1993). The $\mathrm{CH}_{3}$ radical, which is the precursor for the diamond growth gets added onto the growing face. Therefore, for proper diamond growth, sufficient supply of $\mathrm{H}$ is an essential requirement. The presence of the non-diamond band at higher deposition pressure can be explained in terms of the change in the recombination rate of atomic hydrogen. The filament to substrate distance is also an important experimental parameter. With increase in the deposition pressure, the mean free path of the atomic hydrogen 
reduces. This results in its recombination during its course towards the substrate. This recombination rate increases with the decrease in the mean free path, which indeed is the case with increase in the deposition pressure. When sufficient amount of $\mathrm{H}$ is not present, the etching of the terminal hydride is not complete, which results in some of the $-\mathrm{CH}$ bonds being intact. Such a termination would lead to a discontinuity in the $s p^{3}$ tetrahedral network and leads to interesting atomic arrangements (Zhou et al 1996) in terms of hydrogenated vacancies. According to Zhou et al (1996), the single hydrogen thus introduced gets bonded only to a single neighbour of the carbon vacancy. The remaining two of the four neighbouring carbon atoms form a weak stretched pair

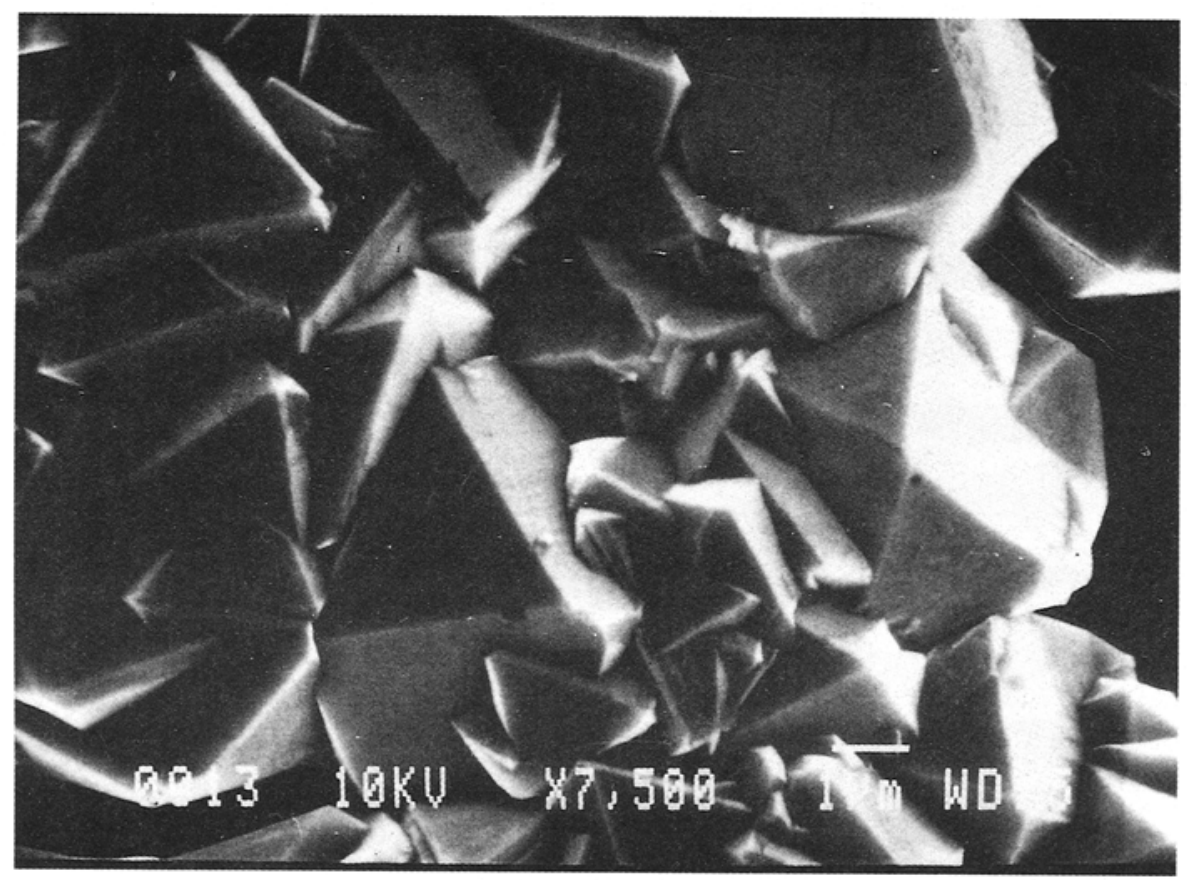

Figure 1. Typical SEM picture of a diamond film deposited by HFCVD technique showing the sharp and clear facets of the diamond grain.

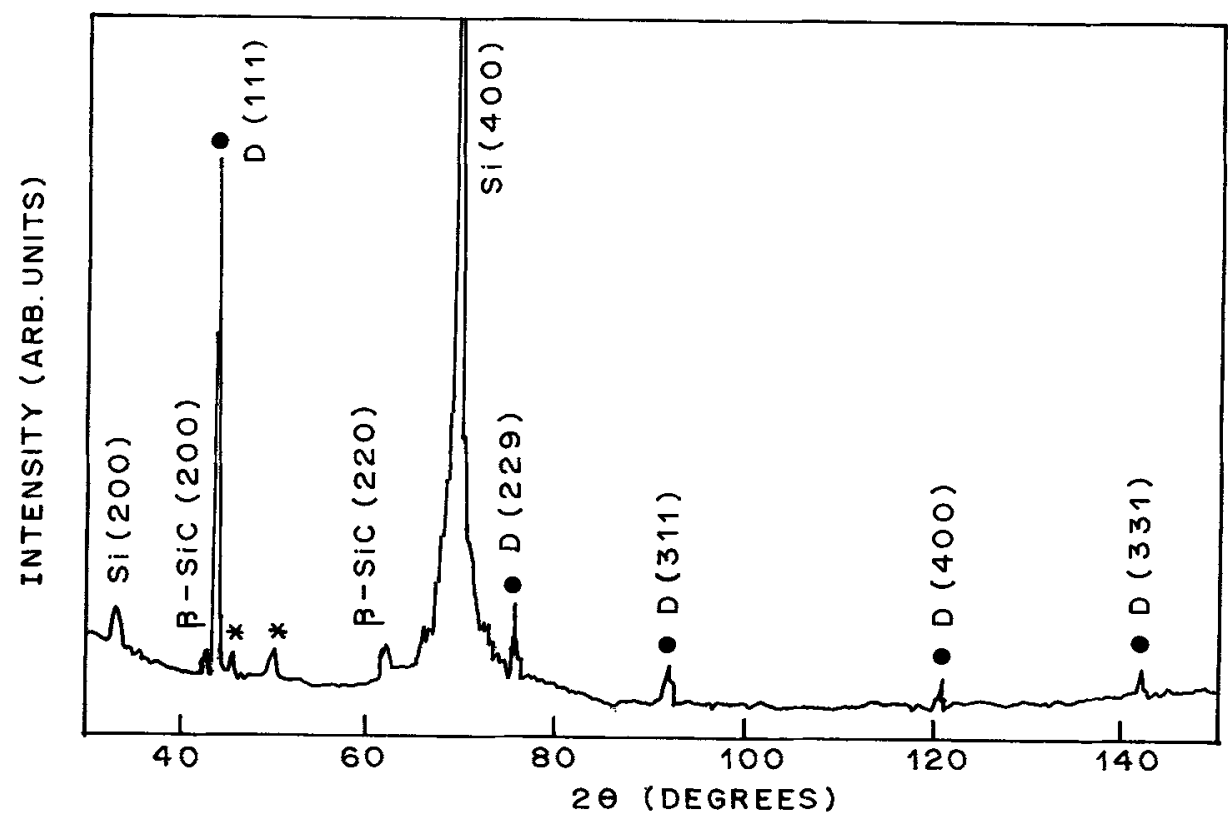

Figure 2. Typical XRD (at $\mathrm{Cu}-\mathrm{K}_{\alpha}$ radiation) pattern of $\mathrm{CVD}$ diamond film. D-denotes XRD peaks corresponding to the reflections from diamond (*denotes unidentified peaks). 


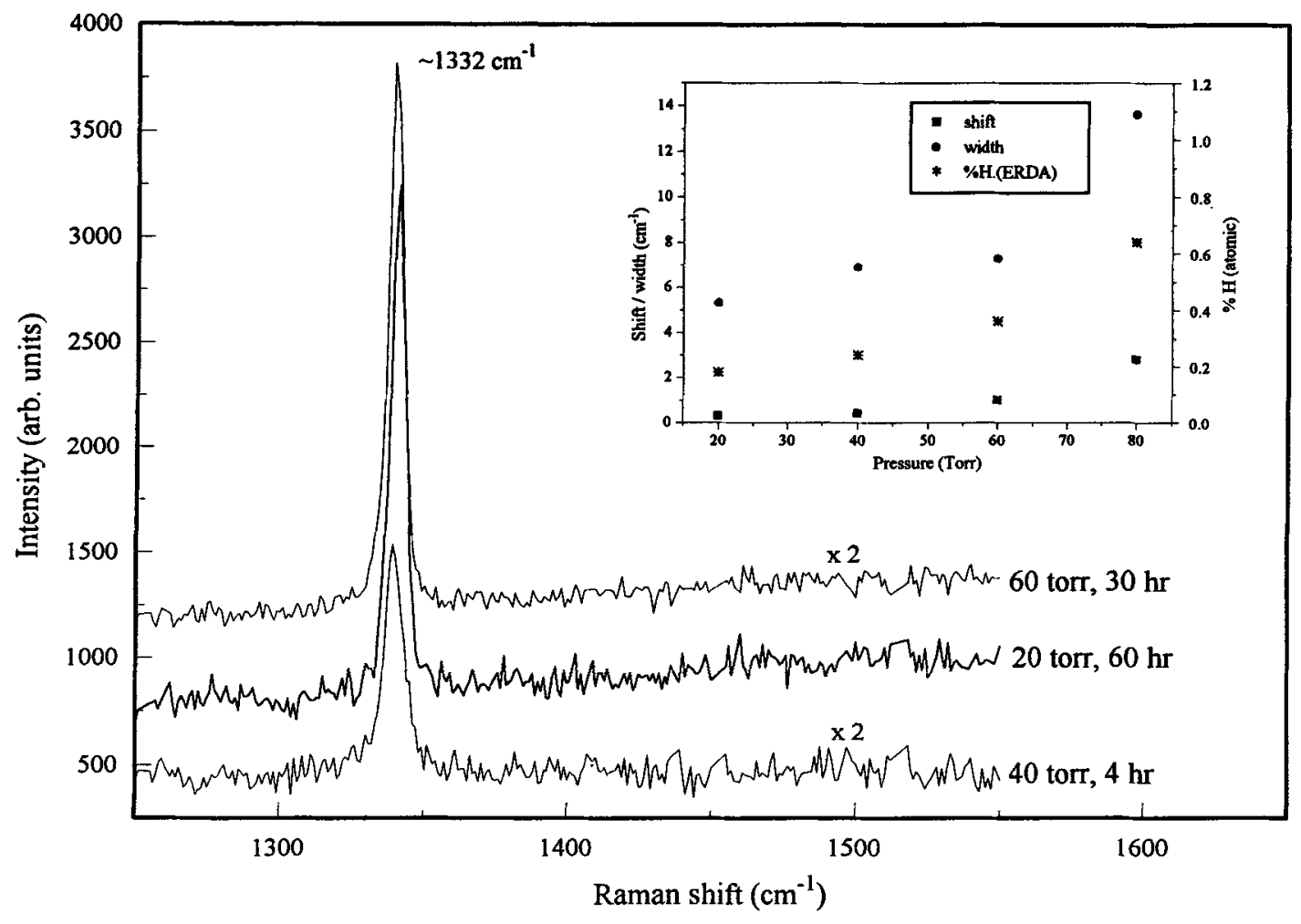

Figure 3. Characteristic diamond peak position in the Raman spectra for samples grown at different deposition pressures (Excitation wavelength $=514.5 \mathrm{~nm}$ of $\mathrm{Ar}^{+}$laser).

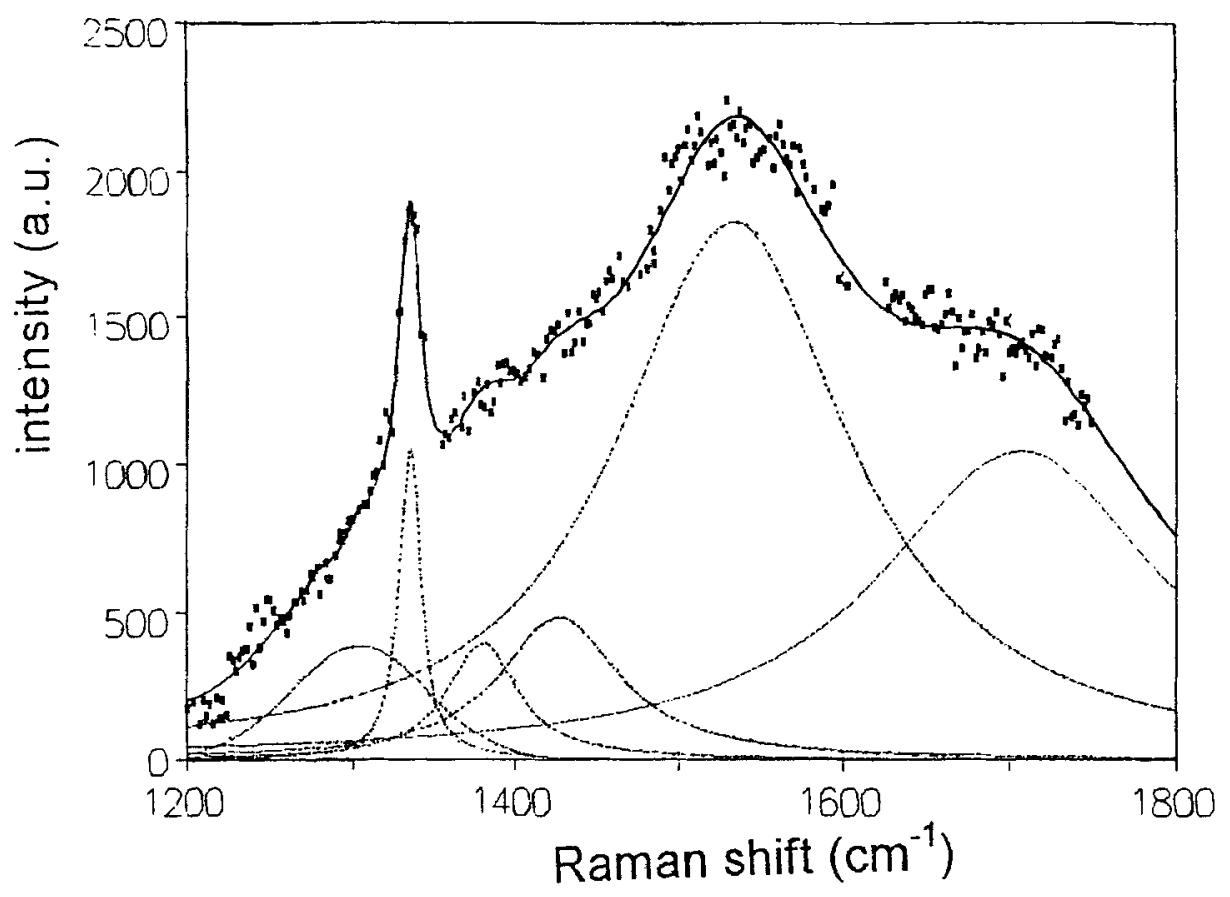

Figure 4. Raman spectra of diamond film grown at 80 torr deposition pressure. The deconvolution of which yields bands at $1380 \mathrm{~cm}^{-1}$ (D-band) and $1427 \mathrm{~cm}^{-1}$ and 1533 $\mathrm{cm}^{-1}$ (G-Band) (excitation wavelength $=488 \mathrm{~nm}$ of $\mathrm{Ar}^{+}$laser). 
Table 1. Assignment of the Raman spectra of the sample grown at 80 Torr pressure.

\begin{tabular}{lccl}
\hline $\begin{array}{l}\text { Peak } \\
\text { no. }\end{array}$ & $\begin{array}{c}\text { Peak position } \\
\left(\mathrm{cm}^{-1}\right)\end{array}$ & $\begin{array}{c}\text { Peak width } \\
\left(\mathrm{cm}^{-1}\right)\end{array}$ & Assignment \\
\hline 1 & $1304 \cdot 0$ & 42 & $*$ \\
2 & $1334 \cdot 7$ & $12 \cdot 3$ & $\begin{array}{l}\text { Crystalline diamond } \\
\left(s p^{3}\right)\end{array}$ \\
3 & 1380 (D-band) & 26 & $\begin{array}{l}\text { Disordered graphite } \\
\left(s p^{2}\right)\end{array}$ \\
4 & 1427 (G-band) & 45 & $\begin{array}{l}\text { Disordered } s p^{3} \text { carbon } \\
(\text { amorphous } t \text {-C) }\end{array}$ \\
5 & 1533 (G-band) & 85 & $\begin{array}{l}\text { Graphite } \\
*\end{array}$ \\
6 & $1706 \cdot 0$ & 103 &
\end{tabular}

*denotes unidentified peaks

of bonds, while the third carbon gets relaxed backwards, with an unpaired spin in its dangling bond orbital. These stretched pair bonds may be responsible for the strain in the sample and are further responsible for the observed shift in the $1332 \mathrm{~cm}^{-1}$ Raman peak at higher deposition pressures. However, the sudden change in the characteristics of the CVD diamond films (as observed by Raman spectroscopy in terms of the shift and the width of $1332 \mathrm{~cm}^{-1}$ peak) in the narrow range $(60-80$ torr) of the deposition pressure, implies that there is an interesting phenomenon occurring, which needs to be investigated. Studies in this direction are already in progress.

Evidence for the presence of the unpaired spins in the dangling bond orbitals of the carbon atom in the vicinity of the hydrogenated vacancies (as described above), are also observed by electron paramagnetic resonance (EPR) measurements obtained on our samples (to be reported elsewhere). The bond length, in one case, i.e. between the atom bordering the vacancy and one of the relaxed carbon atoms, decreases, while the bond length between the carbon atom with the dangling bond and the bonded hydrogen atom (bonded to one of the carbon atoms in the vicinity of the vacancy site) increases. The relaxed carbon atoms may form the $s p^{2}$ environment near such a defect. This will also affect the average grain size of the diamond crystallites. According to the confinement model explained by Richter et al (1981), the broadening of the characteristic diamond peak is observed when the particle size reduces. This may be attributed to the observed sudden increase in the width of the $1332 \mathrm{~cm}^{-1}$ band of our Raman signal. An additional reason for the observed broadening may be the local strain, which modifies the crystallographic lattice to the extent that it partially removes the degeneracy of the $T_{2 \mathrm{~g}}$ mode responsible for the $1332 \mathrm{~cm}^{-i}$ Raman peak. Thus the total broadening may be the resultant of the distortion in the tetrahedral symmetry of diamond (as explained above) and the effect of the grain size. The observation of the feature at $1427 \mathrm{~cm}^{-1}$ corresponding to the disordered mode is in confirmation of the above explanation.

The vacancies thus generated are highly mobile at the temperature of the diamond growth $\left(\sim 2300^{\circ} \mathrm{C}\right)$ which leads to vacancy annealing. However, some of the less mobile vacancies will coalesce and form the vacancy aggregates, which is an essential requirement for the diamond to get stabilized over the thermodynamically more stable graphite phase (Bar-Yam and Moustakas 1989). The inclusion of the atomic hydrogen, as explained above is responsible for the observed non-diamond band in our Raman spectra.

This hypothesis, as it may sound simple helps to explain the non-diamond content observed in our sample deposited at higher deposition pressure. Our earlier elastic recoil detection analysis (ERDA) (Sharda et al 1996) results indicate that increase in deposition pressure increases the hydrogen content in the film (Windischmann et al 1991; Mc Namara et al 1992). This is further supported by our Raman spectroscopic studies. The broad bands in the Raman spectra at $1380 \mathrm{~cm}^{-1}$ (D-band) and at $1427 \mathrm{~cm}^{-1}$ and $1533 \mathrm{~cm}^{-1}$ (G-band) indicate the presence of the non-diamond phase. In literature the origin of these bands has been attributed to the presence of highly disordered form of carbon. The shift and the intensity of this band depends on the crystallite size (Yoshikawa 1989). Since the Raman scattering sensitivity of the non-diamond $\left(5 \times 10^{-5} \mathrm{~cm}^{-1} \mathrm{~g}^{-1}\right)$ is much higher than that of the diamond phase $\left(9.1 \times 10^{-7} \mathrm{~cm}^{-1} \mathrm{~g}^{-1}\right.$ ) (Wada et al 1980), it indicates that the average concentration of these non-diamond $s p^{2}$ defects in the films is very low.

\section{Conclusions}

The diamond thin films grown by the HFCVD technique using $0.8 \% \mathrm{CH}_{4}$ in $\mathrm{H}_{2}$ are polycrystalline and show good diamond growth. The XRD patterns of these thin films show reflection from (111), (400) and (311) planes indicating the multiple orientation of the grains. The reflections from the (111) plane being the most intense, indicate that the growth along $\langle 111\rangle$ is the most favoured. These observations are supported by our SEM results. The Raman spectra of these samples show the characteristic $1332 \mathrm{~cm}^{-1}$ diamond peak. The study of the effect of deposition pressure indicates that there is a range from 20 to 60 torr where there is almost no shift in this peak. However, at higher deposition pressures this peak gets shifted by considerable amount, indicating that the diamond lattice is under strain. Increase in the FWHM of the diamond peak with deposition pressure is also observed. The observed broadening in the $1332 \mathrm{~cm}^{-1}$ peak at higher deposition pressure may be a cumulative effect of strain developed due to the introduction of the 
unetched hydride layer and the disorder present due to discontinuity in the $s p^{3}$ network of diamond. As the deposition pressure is increased beyond 60 torr (say 80 torr and above), the effect of hydrogen impurity creeps in, which is indicated by the presence of two bands at $1380 \mathrm{~cm}^{-1}$ (D-band) and $1427 \mathrm{~cm}^{-1}$ and $1533 \mathrm{~cm}^{-1}$ (G-band). This suggests that at a higher deposition pressure, the growth of non-diamond is promoted along with the growth of the diamond phase.

\section{Acknowledgements}

The authors would like to thank Dr A P Roy for his keen interest in this work. One of the authors (MP) would like to acknowledge the help of Rama Pandey and Susy Thomas during the course of this work.

\section{References}

Angus J C and Haymann C C 1988 Science 241913 Backmann P K and Messier R 1989 C \& EN 24
Bar-Yam Y and Moustakas T D 1989 Nature 342786 Eversole W G 1962 US Patents 3,030,187 and 3,003,188

Fanciulli M and Moustakas T D 1993 Phys. Rev. B48 14982

Liander $\mathrm{H}$ and Lundblad E 1960 Ark. Kemi. 16139

Mc Namara K M, Gleason K K and Robinson C J 1992 J. Vac. Sci. Technol. A10 3143

Richter H, Wang Z P and Ley L 1981 Solid State Commun. 39625

Sharda T, Misra D S and Awasthi D K 1996 Vacuum 471259

Schadler L S and Galiotis C 1995 Int. Mater. Rev. 40116

Spear K E 1989 J. Am. Ceram. Soc. 72171

Von Kaenel Y, Stiegler J, Blank E, Chauvet O, Hellwig Ch and Planmann K 1996 Phys. Status Solidi (a) 154219

Wada N, Gaczi P J and Solin S A $1980 \mathrm{~J}$. Non-Cryst. Solids 35-36 543

Windischmann H, Epps G F, Cong Y and Collins R W 1991 J. Appl. Phys. 692231

Yarbrough W A and Messier R 1990 Science 247688

Yoshikawa M 1989 Mater. Sci. Forum 52-53 365

Zhu W, Stoner B R, Williams B E and Glass J T 1991 Proc. IEEE 79621

Zhou X, Watkins G D, Mc Namara, Rutledge K M, Messiner R P and Sanjay Chawla 1996 Phys. Rev. B54 7881 